\title{
Evidence against hypothalamic-pituitary-adrenal axis suppression in the antidiabetic action of leptin
}

\author{
Gregory J. Morton, Thomas H. Meek, Miles E. Matsen, and Michael W. Schwartz \\ Diabetes and Obesity Center of Excellence, Department of Medicine, University of Washington, Seattle, Washington, USA.
}

\begin{abstract}
Leptin administration restores euglycemia in rodents with severe insulin-deficient diabetes, and recent studies to explain this phenomenon have focused on the ability of leptin to normalize excessive hypothalamic-pituitary-adrenal (HPA) axis activity. Here, we employed a streptozotocin-induced rat model (STZ-DM) of uncontrolled insulin-deficient diabetes mellitus (UDM) to investigate the contribution of HPA axis suppression to leptin-mediated glucose lowering. Specifically, we asked if HPA axis activation is required for diabetic hyperglycemia, whether HPA axis normalization can be achieved using a dose of leptin below that needed to normalize glycemia, and if the ability of leptin to lower plasma glucocorticoid levels is required for its antidiabetic action. In STZ-DM rats, neither adrenalectomy-induced (ADX-induced) glucocorticoid deficiency nor pharmacological glucocorticoid receptor blockade lowered elevated blood glucose levels. Although elevated plasma levels of corticosterone were normalized by i.v. leptin infusion at a dose that raises low plasma levels into the physiological range, diabetic hyperglycemia was not altered. Lastly, the potent glucose-lowering effect of continuous intracerebroventricular leptin infusion was not impacted by systemic administration of corticosterone at a dose that maintained elevated plasma levels characteristic of STZ-DM. We conclude that, although restoring low plasma leptin levels into the physiological range effectively normalizes increased HPA axis activity in rats with uDM, this effect is neither necessary nor sufficient to explain leptin's antidiabetic action.
\end{abstract}

\section{Introduction}

Leptin's unexpected ability to normalize hyperglycemia in rodent models of uncontrolled diabetes mellitus (uDM) has generated strong research interest. Previous work $(1,2)$ showed that, when administered systemically at doses that achieve supraphysiological plasma levels, leptin fully normalizes diabetic hyperglycemia in rodents despite persistent, severe insulin deficiency. Subsequent studies (3-7) demonstrated that intracerebroventricular (icv) or intrahypothalamic administration of leptin (at doses 10to 100 -fold below those needed when given systemically) also restores euglycemia to rats and mice with $\mathrm{uDM}$ induced by the $\beta$ cell toxin streptozotocin (STZ-DM). Based on these observations, the brain was identified as a likely mediator of leptin's antidiabetic actions, and an insulin-independent glucose-lowering mechanism was implied since the effect occurs despite persistent, severe insulin deficiency $(3,4)$.

Plasma levels of both leptin and insulin decline rapidly and severely following STZ administration in rodents (8), raising the possibility that diabetic hyperglycemia is a consequence of leptin deficiency as well as insulin deficiency. If so, simply normalizing plasma leptin levels should substantially improve glycemia in STZ-DM. In an early test of this hypothesis, leptin was administered s.c. to rats with STZ-DM at a dose that achieves physiological leptin replacement $(9,10)$, allowing those manifestations of $\mathrm{uDM}$ that are attributable to leptin deficiency to be distinguished from those that are not. This work demonstrated that, although

Conflict of interest: The authors have declared that no conflict of interest exists. Submitted: May 8, 2015; Accepted: September 28, 2015.

Reference information: J Clin Invest. 2015;125(12):4587-4591. doi:10.1172/JCI82723. several key behavioral and neuroendocrine responses to $\mathrm{uDM}$ were ameliorated by physiological leptin replacement (including hyperphagia, hyperglucagonemia, suppression of the hypothalamic-pituitary-thyroid [HPT] axis, and activation of the hypothalamic-pituitary-adrenal [HPA] axis), the degree of hyperglycemia was not significantly affected (9-11). Although leptin deficiency is required for several behavioral and neuroendocrine manifestations of uncontrolled STZ-DM, it does not explain the associated hyperglycemia. It is relevant to the current work that, since physiological leptin replacement normalized elevated plasma corticosterone levels in this experiment without reversing diabetic hyperglycemia, the effect of leptin to normalize the HPA axis seems unlikely to fully explain its antidiabetic action (9). This conclusion is consistent with earlier work, demonstrating that neither glucocorticoid deficiency induced by adrenalectomy (ADX) $(12,13)$, nor liver-specific glucocorticoid-receptor deletion (14), prevents hyperglycemia in the STZ-DM model.

Recent work by Shulman and colleagues suggests that, in contrast with these earlier findings, physiological leptin replacement does in fact normalize glycemia in rats at the onset of STZ-DM and that this effect can be fully explained by HPA axis suppression (15). This conclusion was based on evidence that, in UDM, HPA axis activation drives lipolysis, generating substrate needed for increased hepatic glucose production (HGP) that, in turn, drives hyperglycemia. Since diabetes-associated activation of the HPA axis is a consequence of leptin deficiency, it follows that physiological leptin replacement is sufficient to prevent both the increase of HGP and the associated hyperglycemia. Buttressing this conclusion was evidence that the glucocorticoid receptor antagonist mifepristone mimics the effect of leptin to normalize hyperglyce- 
A

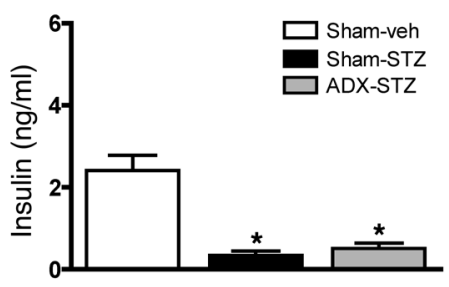

\section{C}

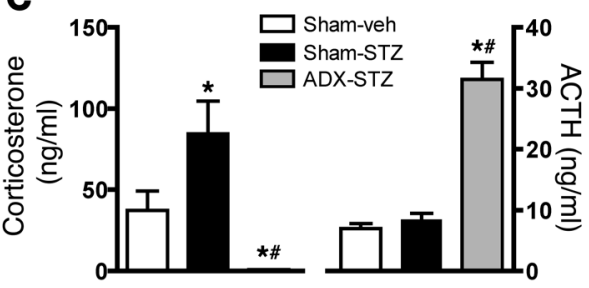

B

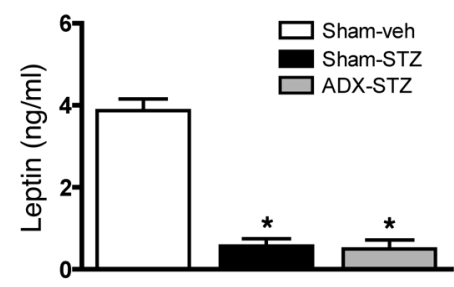

D

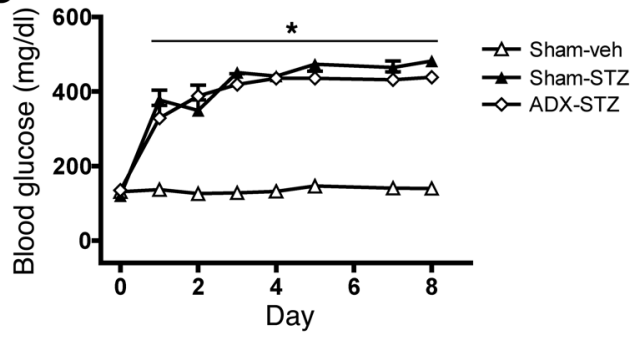

Figure 1. Hypercorticosteronemia is not required for hyperglycemia in STZ-DM. (A-D) Three-hour fasted plasma insulin (A) and leptin (B), and corticosterone and ACTH levels (C) obtained mid-light cycle (13:00), and daily blood glucose levels (D) in the fed state at 09:00 in nondiabetic, sham-operated controls (Sham-veh) and in STZ-diabetic rats that were either sham-operated (Sham-STZ) or underwent surgical ADX (ADX-STZ) ( $n=7-12 /$ group). Data represent mean \pm SEM. Data were analyzed by 1-way ANOVA with a least significant difference post hoc test. ${ }^{*} P<0.05$ vs. Sham-veh; ${ }^{\#} P<0.05$ vs. Sham-STZ.

mia in rats with STZ-DM, while leptin's glucose-lowering effect was blocked by systemic administration of corticosterone (the principal glucocorticoid in rodents) at a dose that achieves the elevated plasma levels characteristic of untreated STZ-DM (15). Together, these findings imply that, in uDM, hyperglycemia is dependent on HPA axis activation that results from leptin deficiency, a conclusion at odds with earlier work.

To further assess the relationship between HPA axis activity and leptin-mediated glucose lowering in rats with STZ-DM, we asked whether (i) HPA axis activation is required for diabetic hyperglycemia, (ii) normalization of the HPA axis can be achieved using a dose of leptin below that needed to normalize glycemia, and (iii) the ability of leptin to lower plasma glucocorticoid levels is required for its glucose-lowering action.

\section{Results and Discussion}

If the effect of leptin to normalize diabetic hyperglycemia is mediated by HPA axis suppression, corticosterone deficiency induced by other means should have a similar salutary effect. As a first test of this hypothesis, we investigated whether corticosterone deficiency induced by ADX ameliorates hyperglycemia in rats with STZ-DM. We found that, although ADX was associated with the expected decrease of plasma corticosterone and increase of plasma adrenocorticotropic hormone (ACTH) levels, the magnitude of hyperglycemia was not affected relative to sham-operated controls (Figure 1), consistent with previous studies $(12,13)$.

One limitation of ADX as a model of glucocorticoid deficiency is that the adrenal gland produces several hormones in addition to corticosterone. It is therefore conceivable that corticosterone deficiency induced by ADX did in fact exert a glucose-lowering effect in rats with STZ-DM, but the effect was offset by other, unrelated consequences of ADX. To address this possibility, we investigated whether glucocorticoid-receptor blockade reverses diabetic hyperglycemia using a recently published protocol for administration of the glucocorticoid receptor antagonist mifepristone to rats within the first 24 hours of STZ-DM (15). We found that, as expected, plasma insulin and leptin levels were reduced (Figure 2A) and plasma ACTH levels were increased following mifepristone administration, signifying effective glucocorticoid-receptor blockade (Figure 2B). Yet the degree of hyperglycemia induced by STZ was unaffected by mifepristone treatment in a single-dose, acute paradigm and following repeated daily dosing (Figure 2, C and D), consistent with previous studies (16). These results collectively indicate that normalization of elevated glucocorticoid receptor signaling is unlikely to explain leptin's antidiabetic action in rats with STZ-DM.

To test whether physiological leptin replacement is sufficient to normalize hyperglycemia as well as HPA axis activity, we again employed a recently reported protocol for i.v. leptin administration at a dose that normalizes plasma leptin levels in rats with STZDM (15). As expected, we found that STZ-DM both lowers plasma insulin and leptin levels (Figure 2, E and F) and raises plasma corticosterone levels (Figure $2 \mathrm{G}$ ), yet normalization of plasma corticosterone levels with leptin replacement failed to ameliorate diabetic hyperglycemia (Figure $2 \mathrm{H}$ ), consistent with earlier reports $(9,10)$. In rats with uDM, therefore, physiological leptin replacement is sufficient to normalize elevated HPA axis activity, but not hyperglycemia, as discussed in a recent review (17).

To determine whether maintenance of elevated plasma corticosterone levels blocks the effect of icv leptin administration to normalize glycemia, we employed a study design in which plasma levels of corticosterone were clamped at either a normal level or at the higher level typical of rats with STZ-DM (Figure 3) (18). All animals underwent ADX and, subsequently, each received (i) a pellet containing a dose of corticosterone designed to maintain plasma corticosterone levels within the physiological range, (ii) STZ to induce $u D M$, (iii) icv leptin at a dose ( $3 \mu \mathrm{g} / \mathrm{d})$ known to normalize glycemia, and (iv) either an additional corticosterone pel- 
A
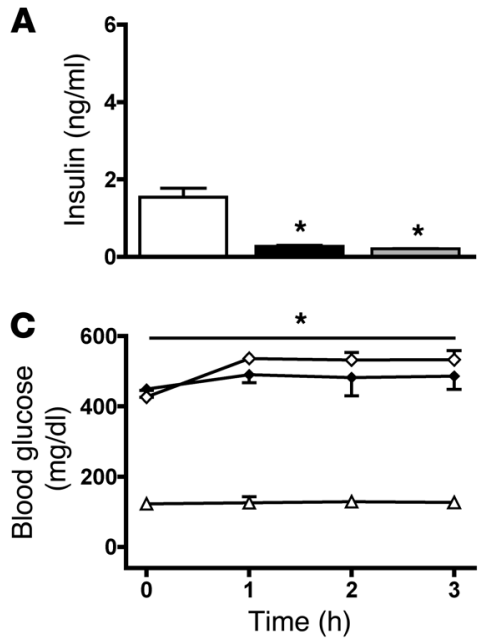

$\mathbf{E}$
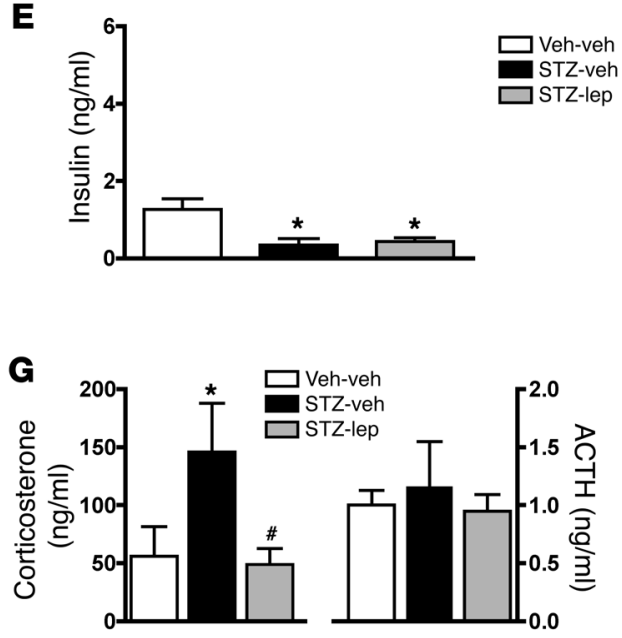

B
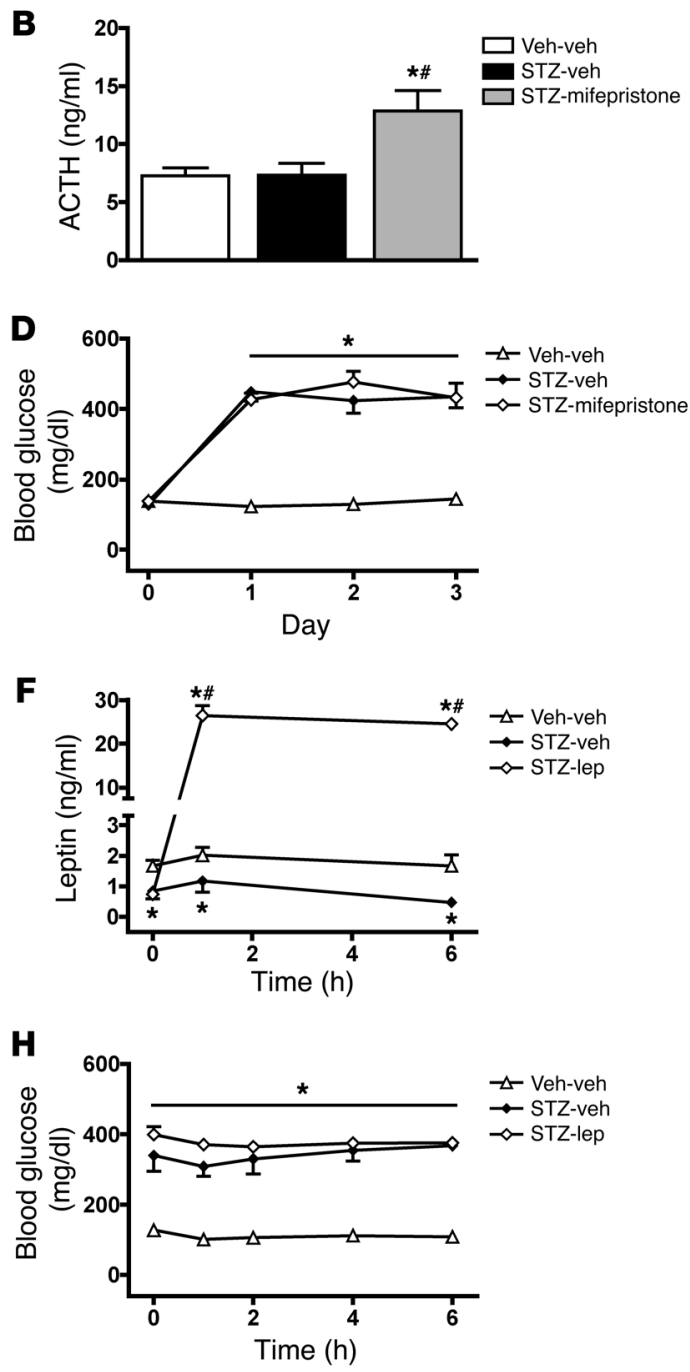

Figure 2. Hyperglycemia in rats with STZ-DM is not reversed by either glucocorticoid receptor blockade or physiological leptin replacement. (A-C) Overnight-fasted plasma insulin (A) and ACTH levels (B) obtained at 12:00 and fasted blood glucose levels (C) over a 3-hour period in either nondiabetic (Veh-veh) or STZ-diabetic rats (within the first 24 hours following STZ) after i.p. administration of either vehicle (STZ-veh) or the glucocorticoid receptor antagonist, mifepristone (STZ-mifepristone; $40 \mathrm{mg} / \mathrm{kg}$ ) ( $n=6-10 /$ group). (D) Daily-fed blood glucose levels (12:00) 3 hours following daily i.p. administration of either vehicle or mifepristone (40 mg/kg) ( $n=6-10 /$ group). (E-H) Overnight-fasted arterial plasma insulin (E) and leptin levels (F), as well as 21-hour fasted plasma corticosterone and ACTH levels (G) obtained at 15:00 and fasted blood glucose levels (H) over a 6-hour period from nondiabetic (Veh-veh) and STZ-diabetic rats (within the first 24 hours following STZ) during which either vehicle (STZ-veh) or leptin (STZ-lep; $150 \mu \mathrm{g} / \mathrm{kg}$ total) was infused i.v. at a dose designed to raise low plasma leptin levels into the high-normal range ( $n=6-8 /$ group). Data represent mean \pm SEM. Data were analyzed by 1 -way ANOVA with a least significant difference post hoc test. ${ }^{*} P<0.05$ vs. Veh-veh; ${ }^{*} P<0.05$ vs. STZ-veh.

let to raise corticosterone levels into the range observed in intact rats with STZ-DM or no pellet (sham-operation) to keep levels in the normal physiological range (Figure 3B). As expected, plasma corticosterone levels were higher (diabetes range) and ACTH levels were lower in the group receiving the additional corticosterone pellet (Figure 3, C and D). Nevertheless, diabetic hyperglycemia was fully normalized by icv leptin infusion, irrespective of whether high or normal plasma corticosterone levels were maintained (Figure 3D). Normalization of plasma corticosterone levels, therefore, is not required for leptin's antidiabetic action.

Our results collectively indicate that (i) HPA axis activation is not required for diabetic hyperglycemia, (ii) normalization of the HPA axis can be achieved using a dose of leptin below that needed to normalize glycemia, and (iii) the ability of leptin to lower plasma glucocorticoid levels is not required for its glucoselowering action. Thus, the effect of leptin to normalize elevated HPA axis activity in rats with STZ-DM is neither necessary nor sufficient to explain how it normalizes glycemia. While these findings are consistent with and extend several previous studies $(1,9,10$, $12,13)$, they are inconsistent with recent evidence from Shulman and colleagues (15) showing that diabetic hyperglycemia is normalized by physiological leptin replacement and that suppression of the HPA axis mediates this effect. Although we endeavored to replicate protocols employed by the Shulman group (15) in several instances, the outcomes do not agree, and a compelling explanation for this discrepancy is not immediately apparent.

A relevant difference in experimental approach is that our studies were conducted in adult male Wistar rats, whereas the 

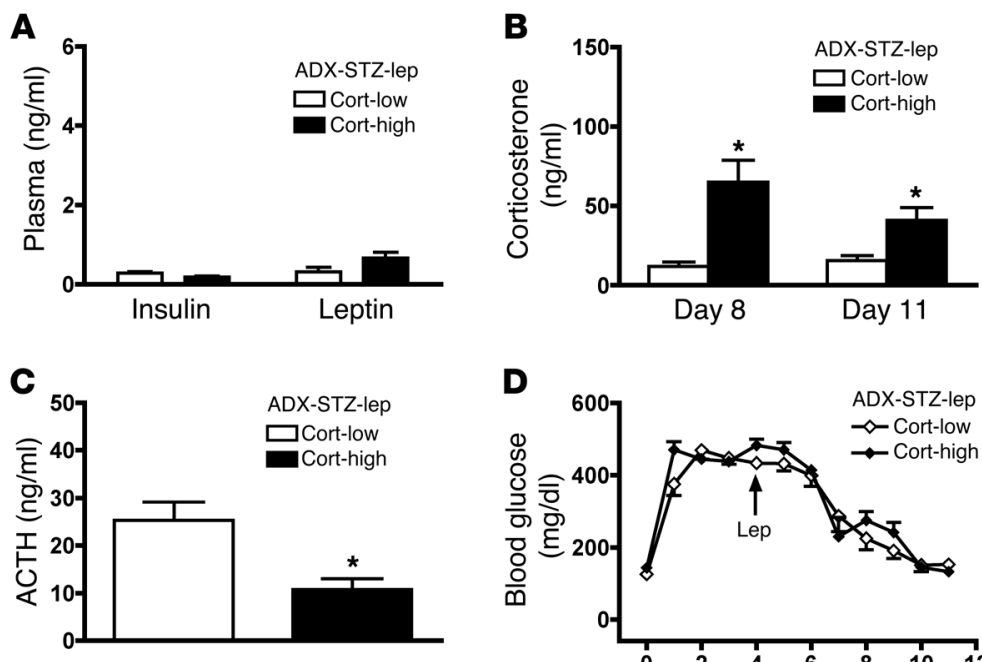

Figure 3. Suppression of hypercorticosteronemia is not required for leptin's antidiabetic effects in STZ-DM. (A-D) Three-hour fasted plasma insulin and leptin (A), corticosterone (B) and ACTH levels (C) obtained mid-light cycle (13:00), and (D) daily-fed blood glucose levels in surgical ADX (ADXSTZ) diabetic rats that received both continuous icv infusion of leptin (beginning on day 4; ADX-STZ-lep), and either a low dose (physiological replacement; $35 \mathrm{mg}$ pellet; Cort-low) or a high dose of corticosterone s.c. (to raise plasma corticosterone levels into the range of rats with STZ-DM; $2 \times 35 \mathrm{mg}$ pellet; Cort-high) ( $n=8-11 /$ group). Data represent mean \pm SEM. Data were analyzed by a 2-tailed, unpaired Student's $t$ test. ${ }^{*} P<0.05$ vs. Cort-low.
Shulman group (15) employed Sprague-Dawley rats, and background strain differences could have been a factor. Another possibility is that the protocol employed by Shulman and colleagues may be associated with a relatively higher degree of stress and hence greater dependence on HPA axis activation to maintain HGP. This stress can potentially be traced to the higher dose of STZ they employed (70 mg/kg/i.v.), in combination with the performance of detailed in vivo metabolic analysis within 24 hours of STZ administration and a prolonged fast. Future investigation is warranted to address this possibility and whether differences in laboratory environment, rat strain, rates of lipolysis, and/or mediators of cellular stress induced by STZ administration or other experimental variables might explain the divergent outcomes. Nevertheless, it is important to determine whether the observations by Shulman and colleagues (15) can be translated across strains and species of animals, including nonhuman primates and ultimately humans, in models with established type 1 diabetes.

Our findings also point to the need for additional studies to delineate the mechanism(s) underlying leptin's antidiabetic action. Clearly, leptin inhibits a number of responses that drive hyperglycemia in the setting of $\mathrm{uDM}$ - including HPA axis activation, hyperglucagonemia, and hyperphagia - but none of these individual effects appears to fully explain leptin's antidiabetic action. In addition to our current analysis of the role of the HPA axis, we recently reported that, although increased glucagon signaling is required for ketosis in rats with STZ-DM, it is not required for hyperglycemia (19). Moreover, physiological leptin replacement normalizes elevated plasma levels of glucagon, but not glucose, in rats with STZ-DM (9). The effect of leptin to normalize elevated glucagon levels in this setting, therefore, does not appear to explain its ability to restore euglycemia, in agreement with data from the Shulman group (15). Similarly, numerous pair-feeding studies indicate hyperphagia is neither necessary nor sufficient for hyperglycemia in this setting $(1-4,7,9,10)$. We note that although the STZ-DM rat model does not recapitulate the pathogenesis of either type 1 or type 2 diabetes in humans, it is nevertheless useful for exploring interactions between leptin and the HPA axis in the setting of diabetes induced by severe, acute insulin deficiency.

We infer from both our current results and from previously published work that, to reverse diabetic hyperglycemia, leptin must simultaneously normalize many distinct behavioral and neuroendocrine responses, and that reversing just one of these (e.g., HPA axis activation) is insufficient. A parallel can be seen in the diverse and redundant nature of the counter-regulatory response to hypoglycemia - because many mechanisms contribute, ablation of any individual component is insufficient to disrupt effective counter-regulation (20). Our data also suggest that, for leptin action to fully restore euglycemia in rats or mice with established diabetes, supraphysiological leptin levels are required (see ref. 17 for a more detailed discussion) and that the underlying mechanism requires leptin exposure for several days $(1-5,7)$.

Our findings have implications beyond leptin-mediated normalization of diabetic hyperglycemia. For example, if HPA axis activation is not needed for hyperglycemia in rats with STZ-DM, as our data suggest, suppression of the HPA axis would seem unlikely to explain the antidiabetic effect of other therapeutic interventions. Among these are peptides in the fibroblast growth factor (FGF) family, 2 of which were recently reported to reverse hyperglycemia in rats with STZ-DM via HPA axis suppression (21). The hypothesis that HPA axis suppression is sufficient to ameliorate diabetic hyperglycemia is also inconsistent with decades of clinical experience that have yet to link HPA axis activation to the pathogenesis of hyperglycemia in human diabetes. Additional experiments are therefore required for a more comprehensive understanding of leptin's antidiabetic effect - information that is crucial to ongoing investigation of the brain as a target for diabetes pathogenesis and treatment (17).

\section{Methods}

Detailed information can be found in Supplemental Methods (supplemental material available online with this article; doi:10.1172/ JCI82723DS1).

Statistics. Results are expressed as mean \pm SEM. Statistical analyses were performed using Statistica (version 7.1; StatSoft Inc., Dell 
Software). A 1-way ANOVA with a least significant difference post hoc test was used to compare mean values between multiple groups, and a 2-tailed, unpaired Student's $t$ test was used for 2-group comparisons. In all instances, probability values of less than 0.05 were considered significant.

Study approval. All procedures were performed in accordance with NIH Guidelines for Care and Use of Animals, and approved by the Institutional Animal Care and Use Committee at the University of Washington and at Harlan Laboratories.

\section{Author contributions}

GJM designed research studies, conducted experiments, acquired data, analyzed data, provided reagents, and wrote the manuscript; THM conducted experiments, acquired and analyzed data, and edited the manuscript; MEM conducted experiments and acquired data; and MWS designed research studies, analyzed data, provided reagents, and wrote the manuscript.

\section{Acknowledgments}

This work was supported by NIH grants DK090320, DK101997, DK083042 (to M.W. Schwartz), and DK089056 (to G.J. Morton); by the Nutrition Obesity Research Center (NORC; DK035816); and by the Diabetes and Metabolism Training Grant (F32 DK097859 and T32 DK0007247) at the University of Washington.

Address correspondence to: Michael W. Schwartz, Department of Medicine, University of Washington at South Lake Union, 850 Republican St, N335, Box 358055, Seattle, Washington, 98195, USA. Phone: 206.897.5288; E-mail: mschwart@ u.washington.edu.
1. Chinookoswong N, Wang JL, Shi ZQ. Leptin restores euglycemia and normalizes glucose turnover in insulin-deficient diabetes in the rat. Diabetes. 1999;48(7):1487-1492.

2. Yu X, Park BH, Wang MY, Wang ZV, Unger RH. Making insulin-deficient type 1 diabetic rodents thrive without insulin. Proc Natl Acad Sci US A. 2008;105(37):14070-14075.

3. Fujikawa T, Chuang JC, Sakata I, Ramadori G, Coppari R. Leptin therapy improves insulindeficient type 1 diabetes by CNS-dependent mechanisms in mice. Proc Natl Acad Sci U S A. 2010;107(40):17391-17396.

4. German JP, et al. Leptin activates a novel CNS mechanism for insulin-independent normalization of severe diabetic hyperglycemia. Endocrinology. 2011;152(2):394-404.

5. Hidaka S, et al. Chronic central leptin infusion restores hyperglycemia independent of food intake and insulin level in streptozotocin-induced diabetic rats. FASEB J. 2002;16(6):509-518.

6. Lin CY, Higginbotham DA, Judd RL, White BD. Central leptin increases insulin sensitivity in streptozotocin-induced diabetic rats. Am J Physiol Endocrinol Metab. 2002;282(5):E1084-E1091.

7. Meek TH, et al. Leptin action in the ventromedial hypothalamic nucleus is sufficient, but not necessary, to normalize diabetic hyperglycemia. Endocrinology. 2013;154(9):3067-3076.

8. Havel PJ, et al. Marked and rapid decreases of circulating leptin in streptozotocin diabetic rats: reversal by insulin. Am J Physiol. 1998; 274(5 pt 2):R1482-R1491.

9. German JP, et al. Leptin deficiency causes insulin resistance induced by uncontrolled diabetes. Diabetes. 2010;59(7):1626-1634.

10. Sindelar DK, Havel PJ, Seeley RJ, Wilkinson CW, Woods SC, Schwartz MW. Low plasma leptin levels contribute to diabetic hyperphagia in rats. Diabetes. 1999;48(6):1275-1280.

11. Matsen ME, et al. In uncontrolled diabetes, thyroid hormone and sympathetic activators induce thermogenesis without increasing glucose uptake in brown adipose tissue. Am J Physio Endocrinol Metab. 2013;304(7):E734-E746.

12. Schwartz MW, Strack AM, Dallman MF. Evidence that elevated plasma corticosterone levels are the cause of reduced hypothalamic corticotrophinreleasing hormone gene expression in diabetes. Regul Pept. 1997;72(2-3):105-112.

13. Strack AM, Sebastian RJ, Schwartz MW, Dallman MF. Glucocorticoids and insulin: reciprocal signals for energy balance. Am J Physiol. 1995; 268(1 pt 2):R142-R149.

14. Opherk C, et al. Inactivation of the glucocorticoid receptor in hepatocytes leads to fasting hypoglycemia and ameliorates hyperglycemia in streptozotocin-induced diabetes mellitus. $\mathrm{Mol}$ Endocrinol. 2004;18(6):1346-1353.

15. Perry RJ, et al. Leptin reverses diabetes by sup- pression of the hypothalamic-pituitary-adrenal axis. Nat Med. 2014;20(7):759-763.

16. Zuo ZF, et al. RU486 (mifepristone) ameliorates cognitive dysfunction and reverses the downregulation of astrocytic N-myc downstreamregulated gene 2 in streptozotocin-induced type- 1 diabetic rats. Neuroscience. 2011;190:156-165.

17. Schwartz MW, et al. Cooperation between brain and islet in glucose homeostasis and diabetes. Nature. 2013;503(7474):59-66.

18. Kovács KJ, Földes A, Sawchenko PE. Glucocorticoid negative feedback selectively targets vasopressin transcription in parvocellular neurosecretory neurons. J Neurosci. 2000;20(10):3843-3852.

19. Meek TH, et al. Evidence that in uncontrolled diabetes, hyperglucagonemia is required for ketosis but not for increased hepatic glucose production or hyperglycemia. Diabetes. 2015;64(7):2376-2387.

20. Cryer PE. Glucose counterregulatory hormones: physiology, pathophysiology, and relevance to clinical hypoglycemia. In: LeRoith D, Taylor SI, Olefsky JM, eds. Diabetes Mellitus: A Fundamental And Clinical Text. 3rd ed. Philadelphia, Pennsylvania, USA: Lippincott Williams \& Wilkins; 2004:199-206.

21. Perry RJ, Lee S, Ma L, Zhang D, Schlessinger J, Shulman GI. FGF1 and FGF19 reverse diabetes by suppression of the hypothalamic-pituitaryadrenal axis. Nat Commun. 2015;6:6980. 